\title{
Disseminated Mycobacterium avium complex infection in acquired immunedeficiency syndrome patient
}

\author{
Pradeep Kumar Mada*, Smitha Maruvada, Andrew Stevenson Joel Chandranesan \\ Affiliated to Louisiana State University Health Sciences Center, Shreveport, United States
}

Received: March 27, 2017

DOI: $10.5430 /$ crim.v4n2p42
Accepted: April 16, 2017

URL: https://doi.org/10.5430/crim.v4n2p42

\begin{abstract}
In the United States, the most common non-tuberculous species causing human diseases are slowly growing species; $M y c o b a c$ terium avium complex (MAC) and Mycobacterium kansasii and rapidly growing species; Mycobacterium abscessus. With the advent of highly active antiretroviral therapy and MAC prophylaxis, disseminated MAC disease is seen infrequently. We report a case of 33-Year-old HIV (Human Immunodeficiency Virus), non-compliant patient presented with disseminated MAC disease. Sputum AFB smear, culture, and Bone marrow biopsy revealed Mycobacterium Avium Intracellulare by DNA (Deoxyribonucleic acid) probe. Following confirmation, he was initiated on Clarithromycin, Ethambutol and Rifabutin for one year of duration with follow up as outpatient.
\end{abstract}

Key Words: Disseminated MAC, AIDS complications

\section{INTRODUCTION}

Mycobacterium Avium Complex, also known as Mycobacterium avium-intracellulare complex (MAC), is comprised of two mycobacterial species: Mycobacterium avium and Mycobacterium intracellulare. Disseminated MAC infection primarily occurs in patients who are immune suppressed, such as people with HIV, and patients on cancer chemotherapy. In the United States, MAC is a non-reportable infection.

The Center for Disease Control's data suggests that MAC infection has an estimated incidence of 1 case per 100,000 persons per year, ${ }^{[1]}$ and study from Oregon has estimated MAC pulmonary infection incidence of 5.6 cases per 100,000 population. ${ }^{[2]} \mathrm{MAC}$ is ubiquitous in distribution and is commonly found in aerosolized water, piped water systems, bathrooms, house dust, soil, birds, and farm animals.

Disseminated MAC usually develops in patients with CD4
(Cluster Differentiation) count less than 50 cells per microliter. Disseminated MAC usually presents with non-specific symptoms. It is usually not the primary suspected diagnosis in the differential of a HIV positive person presenting with non-specific symptoms. We present a case of a 33-year-old HIV positive male with disseminated MAC infection.

\section{Case presentation}

A 33-year-old African American male with past medical history of AIDS (Acquired Immune Deficiency Syndrome), anemia, oral thrush, and Generalized Anxiety Disorder presented to the emergency room complaining of diarrhea for approximately one month of duration. He described his diarrhea as watery stools occurring approximately ten times per day of one month duration, with no specific exacerbating or relieving factors. He reported these symptoms are associated with weakness, which started about one week ago, and result-

\footnotetext{
*Correspondence: Pradeep Kumar Mada, MD; Email: pmada@1suhsc.edu; Address: Affiliated to Louisiana State University Health Sciences Center, Shreveport, United States.
} 
ing in several falls. The patient also complained of shortness of breath and anorexia. The patient denied any cough, wheezing, fever, abdominal pain, and burning urination. Patient also reports unintentional weight loss of thirty five pounds over the last six months. His most recent CD4 count was 12 and his HIV viral load was 156,178 copies per milliliter. His antiretroviral regimen was Darunavir plus Ritonavir, and Emtricitabine/Tenofovir. He was also on Azithromycin for MAC prophylaxis and Trimethoprim/Sulfamethoxazole for Pneumocystis jerovici prophylaxis. However, patient has been non-compliant with his medications. His vitals on admission were temperature of 98 degree Fahrenheit, blood pressure 78/58 millimeter of mercury, pulse rate 113 per minute, respiratory rate 25 per minute. His physical examination was within normal limits, except for marked cachexia. Significant Laboratory findings include: hemoglobin of 6.2 grams per deciliter, white blood cell count of 2,900 cells per microliter, and platelet count of 37,000 cells per microliter. Chest radiograph did not reveal any abnormalities. The patient received two units of packed red blood cells which increased his hemoglobin to 9.6 grams per deciliter. He continued to have persistent hypotension and was given intravenous fluids to elevate his blood pressure. Our hospital dietician was consulted to improve his nutritional status.

His stool cultures were negative for any bacteria and negative for Clostridium difficile toxin. Patient's sputum was sent for an AFB (Acid Fast Bacilli) smear. Two out of the three samples were smear positive for Acid Fast Bacilli. While AFB cultures were pending, Computerized Tomography scan of abdomen and pelvis revealed hepatosplenomegaly with very small hypo dense splenic lesions, a small hypo dense/cystic right hepatic lesion, and mesenteric lymphadenopathy (see Figure 1). Computerized Tomography scan of chest showed right lower lobe infiltrates. The presence of weight loss, pancytopenia, hepatosplenomegaly, and lymphadenopathy in his AIDS background made the diagnosis of disseminated Mycobacterium Avium Intracellulare infection more plausible. Bone marrow biopsy was performed and it showed normocellular bone marrow with noncaseating granulomas, and extensive intracellular acid-fast bacilli microorganisms (see Figures 2 and 3). Around the same time, three out of three specimens of sputum AFB culture were positive by culture within one week, and all three were identified as Mycobacterium Avium Intracellulare by DNA probe. He was started on Clarithromycin $500 \mathrm{mg}$ orally twice a day, Ethambutol $800 \mathrm{mg}$ orally daily, and Rifabutin $300 \mathrm{mg}$ orally daily, based on his weight. Trimehtoprim plus Sulfamethoxazole was recommended for prophylaxis until he achieves sustained CD4 count above 200. Patient was not placed on isolation, since person-to-person transmission appears to be uncommon. Adrenal involvement was suspected to be the cause of his sustained hypotension; however cortisol values were within normal limits. The patient was then discharged home with instructions to complete the remaining treatment at home with regular follow-up appointment at the viral clinic. Patient was followed up in viral clinic after two months. He reported that he was compliant to medication; his symptoms were resolved and gained weight. His viral load was undetectable and CD4 count increased to 59. We emphasized the importance of medication compliance, immunization, advised on safe sex practice and scheduled follow up after 2 months.

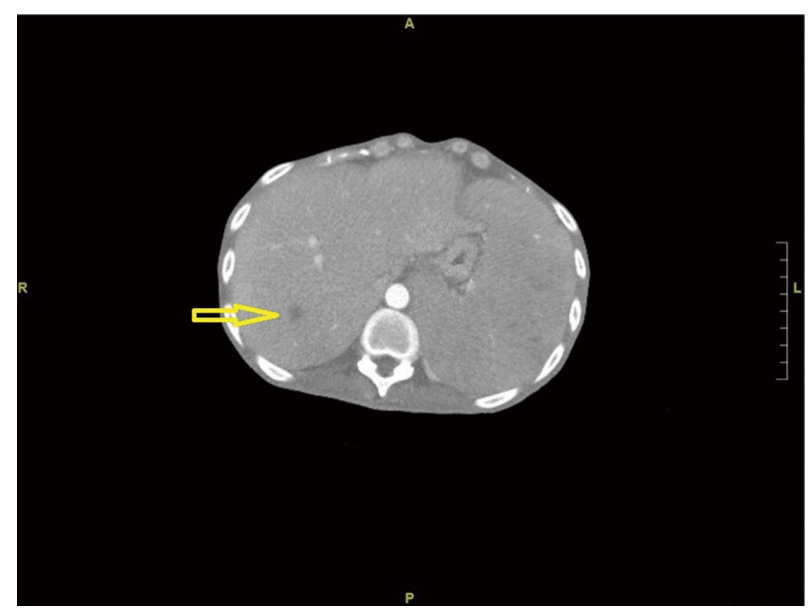

Figure 1. Computerized tomography scan abdomen revealing hepatosplenomegaly with a small right hepatic cystic lesion

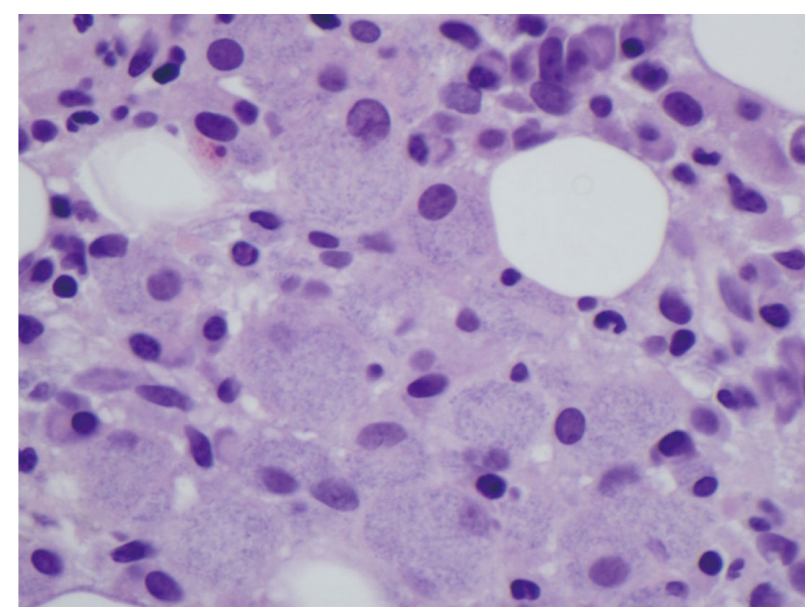

Figure 2. Bone marrow biopsy with hematoxylin and eosin stain on $60 \times$ magnification showing normocellular $(65 \%)$ bone marrow with noncaseating granulomas $(20 \%)$ 


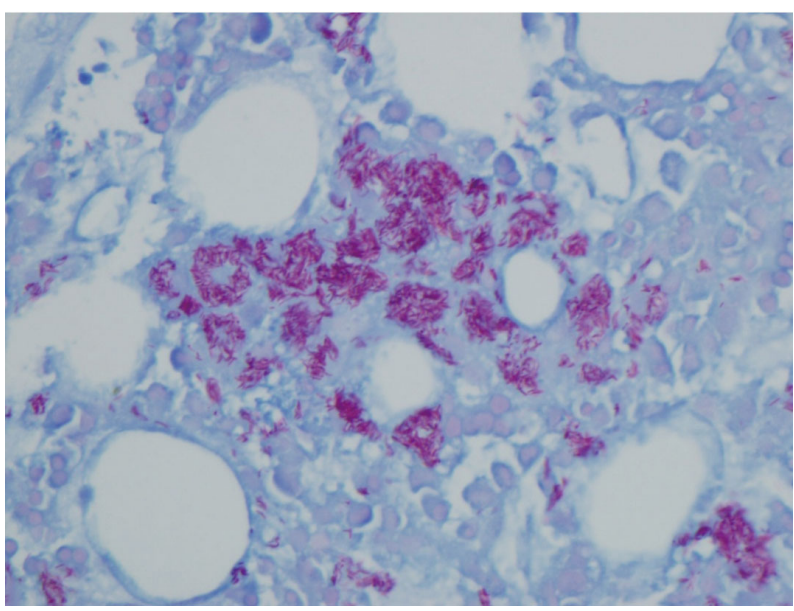

Figure 3. Bone biopsy sample with acid fast bacilli stain on $60 \times$ magnification showing extensive intracellular acid-fast bacilli microorganisms, positive for AFB

\section{Discussion}

MAC infection is most commonly seen among AIDS patients with CD4 counts $<50$ cells per cubic millimeter. It is well established that MAC is associated with a decreased survival in HIV patients. These organisms are ubiquitous in the environment, including water and soil. Infection occurs most commonly through inhalation. The patient presented with severe emancipation, anemia, and other non-specific symptoms. Before the advent of newer macrolide antibiotics and retroviral drugs, disseminated MAC was a fatal infection with a life expectancy of four months. Recent advances in the management have significantly improved the survival and cure rates. ${ }^{[3]}$ Morbidity and mortality associated with disseminated MAC can significantly curtail the initiation of antimicrobial therapy early in the course of the disease. Focal nodular disease presentation has the best prognosis, even though other presentations have a $90 \%$ recovery rate, but a relapse rate of $20 \%$ has been reported. ${ }^{[4]}$ This case emphasizes the importance of including disseminated MAC in the differential in immune compromised patients presenting with non-specific symptoms. Combination drug therapy is required to decrease the risk of drug resistance. Treatment with multiple agents is associated with more rapid clearance of MAC. Duration of treatment usually lasts for at least twelvemonths. Treatment can be safely discontinued three months after attainment of a CD4 cell count of $>100$ cells per cubic millimeter. ${ }^{[5]}$

\section{CONFlicts of InTERest Disclosure}

The authors declare that there is no conflict of interest regarding the publication of this paper.

\section{REFERENCES}

[1] Mycobacterium avium Complex. Centers for Disease Control and Prevention. Available from: http://www.cdc.gov/ncidod/dbm d/diseaseinfo/mycobacteriumavium_t.htm

[2] Cassidy PM, Hedberg K, Saulson A, et al. Nontuberculous mycobacterial disease prevalence and risk factors: A changing epidemiology Clinical Infectious Diseases. 2009; 49(12): e124-9. PMid:19911942 https://doi.org/10.1086/648443

[3] Karakousis PC, Moore RD, Chaisson RE. Mycobacterium avium complex in patients with HIV infection in the era of highly active antiretroviral therapy. Lancet Infectious Disorders. 2004; 4(9): 557-65. https://doi.org/10.1016/S1473-3099(04)01130-2
[4] Field SK, Fisher D, Cowie RL. Mycobacterium avium complex pulmonary disease in patients without HIV infection. Chest. 2004; 126(2): 566-81. PMid:15302746 https ://doi .org/10.1378/ch est.126.2.566

[5] Panel on Opportunistic Infections in HIV-Infected Adults and Adolescents. Guidelines for the prevention and treatment of opportunistic infections in HIV-infected adults and adolescents: Recommendations from the Centers for Disease Control and Prevention, the National Institutes of Health, and the HIV Medicine Association of the Infectious Diseases Society of America. Available from: http://aidsin fo.nih.gov/contentfiles/lvguidelines/adult_oi.pdf 